CNS Spectrums (2019), 24, 616-627. (C) Cambridge University Press 2019. This is an Open Access article, distributed under the terms of the Creative Commons Attribution licence (http://creativecommons.org/licenses/by/4.0/), which permits unrestricted re-use, distribution, and reproduction in any medium, provided the original work is properly cited.

\title{
Long-term functioning outcomes are predicted by cognitive symptoms in working patients with major depressive disorder treated with vortioxetine: results from the AtWoRC study
}

\author{
Pratap Chokka, ${ }^{\text {* }}$ (1) Joanna Bougie, ${ }^{2}$ Jean Proulx, ${ }^{2}$ \\ Anders Holmegaard Tvistholm ${ }^{3}$ and Anders Ettrup ${ }^{4}$
}

\footnotetext{
${ }^{1}$ Department of Psychiatry, Grey Nuns Community Hospital, Edmonton, Canada

${ }^{2}$ Medical and Regulatory Affairs, Lundbeck Canada Inc., Montreal, Canada

${ }^{3}$ Department of Biostatistics, H. Lundbeck A/S, Valby, Denmark

${ }^{4}$ Medical Affairs, H. Lundbeck A/S, Valby, Denmark
}

Objective. AtWoRC (Assessment in Work productivity and the Relationship with Cognitive symptoms) was an interventional, open-label, Canadian study (NCT02332954) designed to assess the association between cognitive symptoms and workplace productivity in working patients with major depressive disorder (MDD) receiving vortioxetine.

Methods. Eligible patients with MDD received vortioxetine $(10-20 \mathrm{mg} /$ day $)$ and were assessed over 52 weeks at visits emulating a real-life setting $(\mathrm{n}=199)$. Partial correlation between changes in patient-reported cognitive symptoms (20-item Perceived Deficits Questionnaire-Depression; PDQ-D-20) and workplace productivity (Work Limitations Questionnaire; WLQ) was assessed at 12 and 52 weeks. Additional assessments included depression severity, cognitive performance, and patient-reported functioning. Structural equations model (SEM) analyses assessed causal relationships between changes in measures of cognition and functioning over time, adjusted for improvements in depressive symptoms.

Results. Statistically significant improvements in all outcomes from baseline to week 52 were seen in the overall population and both subgroups (first treatment and switch). Response and remission rates were $77 \%$ and $56 \%$, respectively. Improvements in PDQ-D-20 and WLQ productivity loss scores at weeks 12 and 52 were significantly correlated. SEM analyses found patient-rated cognitive symptoms (PDQ-D-20) at weeks 12 and 26 were significantly predictive $(p<0.05)$ of patient-reported functioning (Sheehan Disability Scale) at the subsequent visit. Depression severity and objectively measured cognitive performance did not significantly predict functional outcomes at any timepoint.

Conclusion. These results demonstrate the long-term benefits of vortioxetine treatment in working patients with MDD and emphasize the strong association between cognitive symptoms and functioning in a real-world setting.

Received 2 October 2018; Accepted 28 January 2019; First published 25 February 2019

Key words: Cognitive symptoms, functioning, major depressive disorder, real life, vortioxetine, work productivity.

\footnotetext{
*Address for correspondence: P. Chokka, Grey Nuns Community Hospital, 1100 Youville Drive West, Edmonton, Alberta T6L 5X8, Canada (E-mail: pratapchokka@shaw.ca)

Under the direction of the authors, medical writing assistance was provided by Jennifer Coward of Anthemis Consulting Ltd, funded by H. Lundbeck A/S. All authors were involved at all stages of manuscript development, approved the final version of the manuscript to be published, and agreed to act as guarantors of the work. The authors would like to thank Dr. Guerline Clerzius of Lundbeck Canada Inc. for her valuable input into development of the manuscript. The authors would also like to thank JSS Medical Research, the contract research organization mandated to manage the study, and the following principal investigators at the participating study sites: Dr. Sunny Johnson, Dr. Pratap Chokka, Dr. Paul Latimer, Dr. Brian Ramjattan, Dr. Mark Johnston, Dr. Michael O’Mahony, Dr. Sean Peterson, Dr. William O’Mahony, Dr. Murray Awde, Dr. Michael Csanadi, Dr. Ranjith Chandrasena, Dr. Preston Zuliani, Dr. Peter Turner, Dr. Richard Tytus, Dr. Jeannette Janzen, Dr. Guy Chouinard, Dr. Giuseppe Mazza, Dr. Denis Beaulieu, Dr. Valérie Tourjman, Dr. François Blouin, Dr. Marie-Chantal Ménard, Dr. Benicio Frey, Dr. Hani Iskandar, Dr. Arun Nayar, Dr. Jasmin Belle-Isle, and Dr. Ethel Bellavance. This study was supported by Lundbeck Canada.
} 


\section{Introduction}

Functional impairment is a key consequence of major depressive disorder (MDD). ${ }^{1,2}$ Multiple domains of functioning are typically impaired in patients with MDD, particularly their ability to work and work productivity. ${ }^{3-7}$ Depression has a considerable impact in the workplace worldwide. ${ }^{8}$ Of $\sim 1.3$ million Canadians aged 15-65 years who experienced a depressive episode in 2012 , over 1 million were employed. ${ }^{9}$ Of these, only $17 \%$ were reported to be fully functioning at work; $23 \%$ were unable to work due to their depression, $20 \%$ worked part-time due to their depression, and $40 \%$ worked full-time but with reduced functioning.

The Canadian Network for Mood and Anxiety Treatments (CANMAT) guidelines for the management of MDD state that recovery from depression involves both relief of symptoms and improvement of functioning. ${ }^{10}$ MDD is a multidimensional disease characterized by emotional, physical, and cognitive symptoms, all of which may require assessment and treatment to achieve functional recovery. ${ }^{10-14}$ Systematic reviews have shown that improvement in mood symptoms is only modestly correlated with functional outcomes in MDD. ${ }^{13,15-17}$ Functional impairment can persist in patients with MDD even after remission of mood symptoms, ${ }^{2,18,19}$ and residual functional impairment has been associated with an increased risk of relapse and recurrence of depression. ${ }^{20,21}$

The clinical relevance of cognitive symptoms, including disturbances in attention, memory, processing speed, and executive functioning, and their role in work-related disability is well documented in MDD. ${ }^{16,22-27}$ Compared with depression severity, cognitive symptoms have been reported to account for greater impairment in workplace functioning in patients with MDD. ${ }^{28}$ Treatment of cognitive symptoms may hold the key to achieving functional recovery in MDD; however, the relationship between cognitive symptoms and functional impairment in MDD is not well understood.

Vortioxetine is a multimodal antidepressant approved for the treatment of MDD in adults, which acts as an inhibitor of the serotonin transporter as well as modulating the activity of multiple serotonin receptor subtypes. ${ }^{29,30}$ Vortioxetine has been shown to be effective not only for the treatment of MDD, ${ }^{31,32}$ but also to improve cognitive symptoms in patients with depression. ${ }^{33-38} \mathrm{~A}$ recent meta-analysis showed that vortioxetine also demonstrates efficacy in improving overall functioning and functional remission, as assessed by the Sheehan Disability Scale (SDS), in adults with MDD. ${ }^{39}$

Assessment in Work productivity and the Relationship with Cognitive symptoms (AtWoRC) is an interventional, open-label, real-world study undertaken to examine the association between cognitive symptoms and workplace productivity in working Canadian patients with MDD treated with vortioxetine. The primary analysis of this study showed a statistically significant association between improvements in cognitive symptoms in patients with MDD and workplace productivity after 12 weeks of vortioxetine treatment. ${ }^{40}$ Patients with MDD generally require long-term treatment; current CANMAT guidelines recommend antidepressant treatment continuing for at least 6 months after achieving symptomatic remission and for 2 years or longer in patients with risk factors for recurrence. ${ }^{10}$ However, only limited data are available concerning the long-term real-world effectiveness of antidepressants. This article presents the analysis of the AtWoRC study after 52 weeks of vortioxetine treatment in clinical practice, together with structural equations model (SEM) analyses undertaken to further assess causal relationships between long-term changes in measures of cognition and functioning in working patients with MDD.

\section{Methods}

\section{Study design}

AtWoRC is an interventional, open-label study conducted at 26 sites across Canada, mainly in primary care settings (ClinicalTrials.gov identifier: NCT02332954). The study design and inclusion/exclusion criteria have been reported in detail previously. ${ }^{40}$ Briefly, eligible patients were aged 18-65 years; were in employment (working $\geq 20$ hours/week) or enrolled in full-time post-secondary studies or vocational training; had a current diagnosis of MDD according to Diagnostic and Statistical Manual of Mental Disorders, 5th Edition (DSM- ${ }^{\mathrm{TM}}$ ) criteria $^{1}$ and an investigator-confirmed current major depressive episode of at least 3 months' duration; had a baseline Quick Inventory of Depressive Symptomatology-SelfReport (QIDS-SR) score $\geq 15$ and a baseline 20-item Perceived Deficits Questionnaire-Depression (PDQ-D20 ) score $\geq 30$; and had not previously received vortioxetine. Exclusion criteria included the following: Digit Symbol Substitution Test (DSST) score $>69$ at screening/baseline; current diagnosis or history of mania or hypomania, schizophrenia, or any other psychotic disorder (including MDD with psychotic features), personality disorder, attention-deficit hyperactivity disorder, mental retardation, pervasive development disorder, organic mental disorders, or mental disorder due to a general medical condition (DSM-5 criteria); physical, cognitive, or language impairment of such severity as to adversely affect the validity of the data derived from the patientreported outcomes; current depressive symptoms considered to have been resistant to 2 adequate antidepressant treatments of at least 6 weeks' duration, each at the maximum recommended dose according to Canadian labeling; and previous exposure to vortioxetine. 


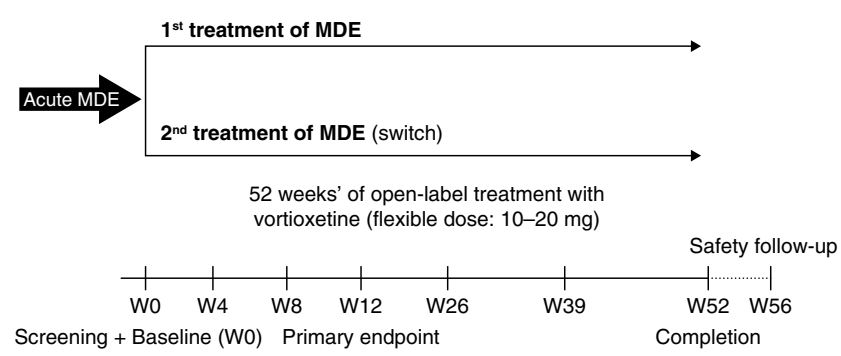

FIGURE 1. Design of the AtWoRC study. MDE, major depressive episode (current episode); W, week.

The study design is shown in Figure 1. All patients received oral vortioxetine $10-20 \mathrm{mg}$ daily (Trintellix ${ }^{\circledR}$, Lundbeck) and were assessed regularly at visits that emulated a naturalistic, real-life setting as closely as possible. Patients were stratified according to whether vortioxetine was their first treatment for the current depressive episode or they were switching to vortioxetine due to an inadequate response to antidepressant treatment of the current episode. The study duration was 52 weeks, with a safety follow-up visit at week 56 . Patients were assessed at visits at baseline and weeks 4, 8, 12, 26 , 39 , and 52 .

Ethical approval was obtained from the necessary committees for each study site, and all patients provided written informed consent for participation.

\section{Study assessments}

The assessment tools used in this study are described in detail in Supplementary Table 1, available online. In brief, depression severity was assessed by patients using the QIDS-SR and by clinicians using the Clinical Global Impressions-Severity and -Improvement scales (CGI-S and CGI-I, respectively). Treatment response was defined as a change in QIDS-SR of $\geq 50 \%$ from baseline. Remission was defined as a QIDS-SR total score $\leq 5$. Cognitive symptoms and performance were assessed by the PDQ-D-20 and the DSST. Functioning and work productivity were assessed by the Work Limitations Questionnaire (WLQ) productivity loss, the SDS, the Work Productivity and Activity Impairment (WPAI) questionnaire, and the 12-item World Health Organization Disability Assessment Schedule 2.0 (WHODAS). Anxiety symptoms were assessed using the Generalized Anxiety Disorder 7-item questionnaire (GAD-7). Safety, tolerability (reporting of adverse events [AEs]), and rate of treatment discontinuation were also assessed.

\section{Statistical analysis}

Sample size calculations have been described in detail previously. ${ }^{40}$ The population for analysis comprised all patients who met the study inclusion criteria and received at least 1 dose of vortioxetine with a valid baseline assessment and at least 1 complete post-baseline visit (full analysis set). Safety and tolerability were evaluated in all enrolled patients who received at least 1 dose of vortioxetine. All efficacy analyses were conducted in the overall population (full analysis set), and for the first-treatment and switch patient groups.

The primary study endpoint was the partial correlation between changes in PDQ-D-20 and WLQ productivity loss scores at week 12 ; this was also assessed at week 52 . The correlation between the change from baseline to weeks 12 and 52 in PDQ-D-20 and WLQ productivity loss scores was described by the partial Pearson's correlation coefficient adjusted for age, sex, baseline PDQ-D-20, baseline WLQ productivity loss, disease duration, and baseline depression severity (baseline QIDS-SR and CGI-S). Secondary endpoints included change from baseline to weeks 12 and 52 in disease severity (QIDS-SR, CGI-S, and CGI-I), cognitive symptoms and performance (PDQ-D-20 and DSST), work productivity (WLQ productivity loss and WPAI overall impairment), functioning (SDS and WHODAS), and symptoms of anxiety (GAD7 ). Rates of treatment response and remission were also calculated at weeks 12 and 52. For all secondary endpoints, Student's $t$ tests were performed to assess change from baseline and compare between-group differences.

To assess temporal dependence (ie, causality) between changes from baseline in patient-reported cognitive symptoms, functioning, and overall depressive symptomatology, one-lag SEM analyses were performed, which allowed 4 outcome measures to be predicted by the scores of each outcome at the immediately previous visit (PDQ-D-20, DSST, QIDS-SR, with SDS/WLQ productivity loss in 2 separate models). Standardized regression coefficients (SRC) from the SEM were used to evaluate the strength of each relationship over time, with an SRC having an absolute value $\geq 0.2$ considered indicative of robust associations. The 2 models were estimated with the full information maximum likelihood estimator on the full analysis set using the statistical software SAS version 9.4 (SAS Institute Inc., Cary, NC, USA). The full information maximum likelihood estimator produces unbiased estimates in the event of missing data, under the assumption that the data are missing at random. For model evaluation, the root mean square error of 
TABLE 1. Baseline patient demographics, employment status, and clinical characteristics*

\begin{tabular}{|c|c|c|c|}
\hline Characteristic & First treatment $(n=107)$ & Switch $(n=112)$ & All treated $(n=219)^{+}$ \\
\hline Age (years), mean $(S D)^{\ddagger}$ & $38.9(12.7)$ & $42.6(12.0)$ & $40.8(12.5)$ \\
\hline Female, \% (n) & $70.1(75)$ & $68.8(77)$ & $69.4(152)$ \\
\hline Caucasian, \% (n) & $93.5(100)$ & $94.6(106)$ & $94.1(206)$ \\
\hline \multirow[t]{2}{*}{ Time since MDD diagnosis (years), mean $(\mathrm{SD})^{\ddagger}$} & $5.6(6.5)$ & $11.0(11.3)$ & $8.4(9.6)$ \\
\hline & First treatment $(n=97)$ & Switch $(n=102)$ & Full analysis set $(n=199)$ \\
\hline \multicolumn{4}{|l|}{ Highest level of education, \% (n) } \\
\hline High school or less & $37.1(36)$ & $40.2(41)$ & $38.7(77)$ \\
\hline College & $40.2(39)$ & $37.3(38)$ & $38.7(77)$ \\
\hline University & $14.4(14)$ & $11.8(12)$ & $13.1(26)$ \\
\hline Post-graduate & $1.0(1)$ & $7.8(8)$ & $4.5(9)$ \\
\hline \multicolumn{4}{|l|}{ Employment type, \% (n) } \\
\hline Employed/independent & $89.7(87)$ & $94.1(96)$ & $92.0(183)$ \\
\hline Full-time vocational & $4.1(4)$ & $1.0(1)$ & $2.5(5)$ \\
\hline Full-time post-secondary student & $6.2(6)$ & $2.9(3)$ & $4.5(9)$ \\
\hline \multicolumn{4}{|l|}{ Occupation, \% (n) } \\
\hline Manufacturing & $5.2(5)$ & $2.9(3)$ & $4.0(8)$ \\
\hline Professional & $8.2(8)$ & $7.8(8)$ & $8.0(16)$ \\
\hline Service staff & $9.3(9)$ & $13.7(14)$ & $11.6(23)$ \\
\hline Sales & $12.4(12)$ & $10.8(11)$ & $11.6(23)$ \\
\hline Clerical & $12.4(12)$ & $14.7(15)$ & $13.6(27)$ \\
\hline Healthcare & $9.3(9)$ & $7.8(8)$ & $8.5(17)$ \\
\hline Agriculture & $0(0)$ & $1.0(1)$ & $0.5(1)$ \\
\hline Construction & $6.2(6)$ & $3.9(4)$ & $5.0(10)$ \\
\hline Other & $27.8(27)$ & $30.4(31)$ & $29.1(58)$ \\
\hline \multicolumn{4}{|c|}{ Clinical characteristics, mean (SD) scores at baseline (week 0) } \\
\hline PDQ-D-20 & $49.8(12.1)$ & $49.6(12.1)$ & $49.7(12.1)$ \\
\hline QIDS-SR & $18.7(2.6)$ & $18.1(2.6)$ & $18.4(2.6)$ \\
\hline GAD- $7^{\ddagger}$ & $15.6(4.7)$ & $14.1(4.9)$ & $14.8(4.8)$ \\
\hline CGI-S & $4.1(0.6)$ & $4.1(0.5)$ & $4.1(0.5)$ \\
\hline WLQ, \% productivity loss & $13.2(4.6)$ & $13.6(4.5)$ & $13.4(4.6)$ \\
\hline WPAI, \% overall impairment & $66.6(22.9)$ & $69.3(23.6)$ & $68.0(23.3)$ \\
\hline SDS & $21.0(4.7)$ & $21.0(5.5)$ & $21.0(5.1)$ \\
\hline WHODAS & $21.1(6.8)$ & $21.0(7.9)$ & $21.0(7.4)$ \\
\hline DSST (number of correct symbols) & $47.8(11.2)$ & $45.3(11.5)$ & $46.5(11.4)$ \\
\hline Vortioxetine dose at week $52(\mathrm{mg} /$ day), mean (SD) & $14.6(5.0)$ & $15.8(5.2)$ & $15.2(5.1)$ \\
\hline
\end{tabular}

Notes: *For patient demographics, all treated patients were assessed. For employment status and clinical characteristics, patients in the full analysis set were assessed.

'Data for 3 patients were unavailable and therefore not reported at the time of the 12-week primary analysis; information was available at the time of this analysis at week 52 and is included here.

¥Significantly different between groups $(p<0.05)$.

CGI-S, Clinical Global Impression-Severity; DSST, Digit Symbol Substitution Test; GAD-7, 7-item Generalized Anxiety Disorder Scale; MDD, Major Depressive Disorder; PDQ-D-20, 20-item Perceived Deficits Questionnaire-Depression; QIDS-SR, Quick Inventory of Depressive Symptomatology-Self-Report; SD, standard deviation; SDS, Sheehan Disability Scale; WHODAS, 12-item World Health Organization Disability Assessment Schedule 2.0; WLQ, Work Limitations Questionnaire; WPAI, Work Productivity and Activity Impairment.

approximation and comparative fit index were used (good fit generally accepted as $<0.08$ and $>0.90$, respectively). ${ }^{41,42}$ A similar modeling approach was recently used to explore causality between cognitive symptoms, depression severity, and functioning in a longitudinal observational study in European patients with MDD. ${ }^{43}$

\section{Results}

\section{Study population}

The first patient was enrolled in February 2015, and the last patient completed the study in July 2017. Patient disposition is shown in Supplementary Figure 1, available online. A total of 219 patients were enrolled in the study and received at least 1 dose of vortioxetine (107 first treatment, 112 switch). In all, 199 patients attended at least 1 post-baseline visit and were included in the full analysis set (first treatment $\mathrm{n}=97$, switch $\mathrm{n}=102$ ). The mean (SD) daily dose of vortioxetine at week 52 was 15.2 (5.1) $\mathrm{mg}$.

Baseline patient demographics and clinical characteristics are shown in Table 1 . Switch patients were significantly older than first-treatment patients $(42.6$ versus 38.9 years; $p=0.030)$, and had a longer time since first diagnosis of MDD (11.0 versus 5.6 years; $p<0.001)$. 

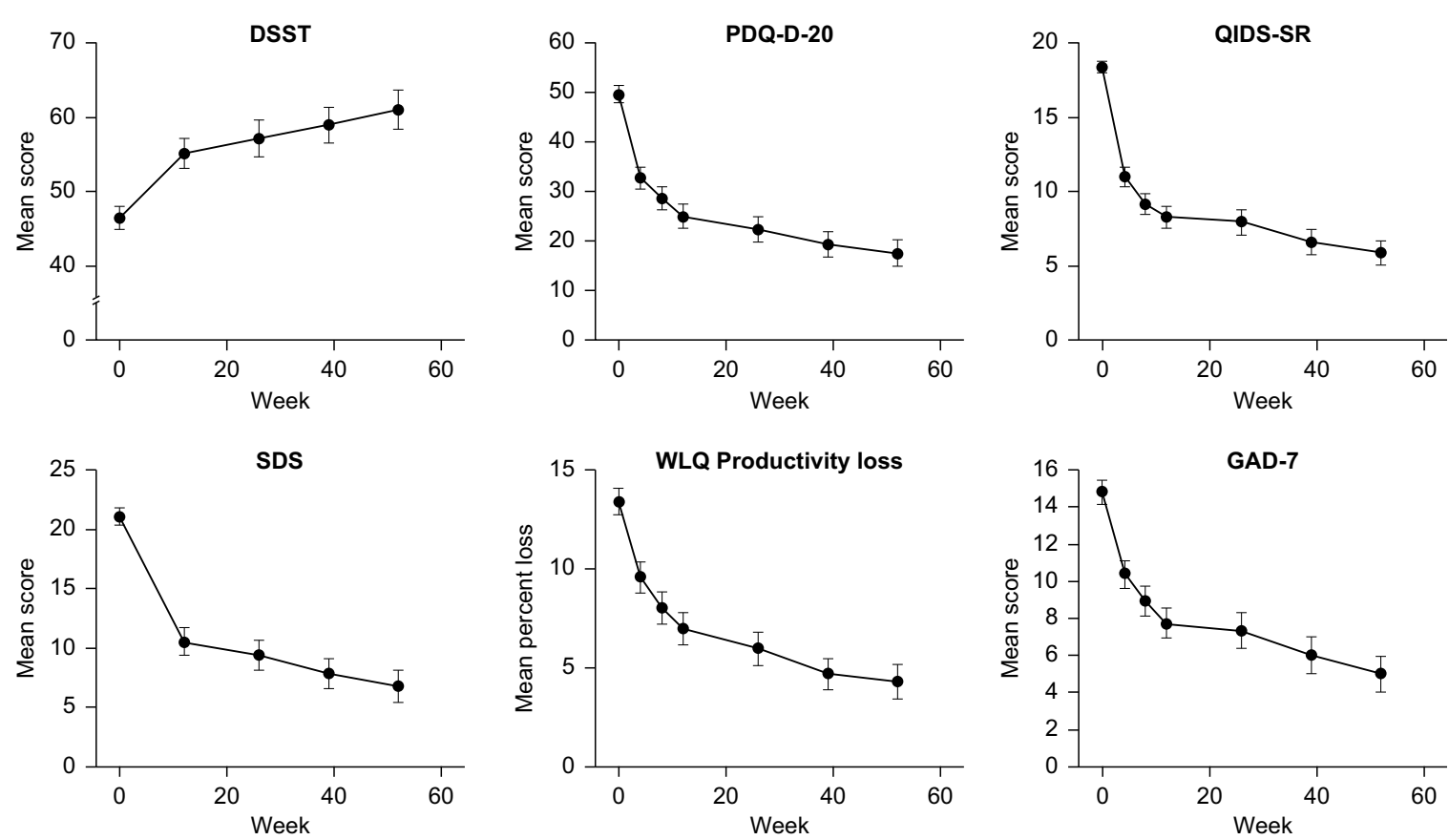

FIGURE 2. Changes in cognitive performance and symptoms, overall depressive symptoms, overall and workplace functioning, and anxiety symptoms over the 52 weeks of vortioxetine treatment. Mean DSST, PDQ-D-20, QIDS-SR, SDS, WLQ productivity loss and GAD-7 scores over the 52 weeks of follow-up are shown; error bars indicate $95 \%$ confidence intervals. Significant improvements $(p<0.001$, paired $t$ test) versus baseline were found for all outcomes at week 52. DSST, Digit Symbol Substitution Test; GAD-7, 7-item Generalized Anxiety Disorder Scale; PDQ-D-20, 20-item Perceived Deficits Questionnaire-Depression; QIDS-SR, Quick Inventory of Depressive Symptomatology-Self-Report; SDS, Sheehan Disability Scale; WLQ, Work Limitations Questionnaire.

At baseline, patients in both groups were acutely depressed, with "severe" scores for cognitive symptoms (PDQ-D-20), overall depressive symptoms (QIDS-SR), anxiety symptoms (GAD-7), and functional impairment (SDS). First-treatment patients had more severe anxiety than switch patients $(p=0.029)$.

\section{Long-term treatment outcomes}

Significant improvements in disease severity (QIDS-SR and CGI-S), cognitive symptoms and objective cognitive performance (PDQ-D-20 and DSST), anxiety symptoms (GAD-7), work productivity (WLQ productivity loss and WPAI overall impairment), and functioning (SDS and WHODAS) were observed over the 52 weeks of vortioxetine treatment $(p<0.001$ [paired $t$ test] versus baseline for all outcomes at week 52; Figure 2 and Table 2). No significant differences were reported in mean change from baseline to week 52 between the first-treatment and switch groups for any outcome measure. In all, $77 \%$ of patients achieved treatment response $(71 \%$ in the first-treatment group and $83 \%$ in the switch group), and $56 \%$ of patients achieved remission $(45 \%$ in the first-treatment group and $67 \%$ in the switch group; $p=0.017)$ after 52 weeks of vortioxetine treatment (Figure 3). The percentage of patients reporting missed work days due to depression in the past 3 months was reduced, from $55 \%$ at baseline to $9 \%$ at week 52 (observed cases in the total population). In patients reporting missed work days due to depression, the mean number of work days missed in the past 3 months decreased from 13 days at baseline to 8 days at week 52 .

Individual changes from baseline to week 52 in PDQD-20 total score and WLQ productivity loss suggested an association between these 2 outcomes (Figure 4). In general, patients who had improved cognitive function following treatment with vortioxetine also had improved workplace productivity. For most outcomes, correlations between changes from baseline to week 52 were highly significant; in particular, significant associations were seen between changes in PDQ-D-20 score and changes in all other outcome measures, including GAD-7 score (Table 3). Reduction in GAD-7 scores was observed at 12 weeks and persisted after 52 weeks of vortioxetine treatment, from "severe" anxiety at baseline (mean score, 14.8; 95\% confidence interval [CI], 14.12-15.47) to "mild" anxiety at week $12(7.7 ; 95 \%$ CI, 6.93-8.56) and week 52 (5.0; 95\% CI, 4.04-5.93).

A strong and highly significant association was seen between the changes in PDQ-D-20 and WLQ productivity loss scores assessed by the partial correlation coefficient 
TABLE 2. Change from baseline to week 52 in assessment scores in the full analysis set (observed cases)*

\begin{tabular}{lccc} 
& \multicolumn{3}{c}{ Mean (SD) change } \\
\cline { 2 - 4 } Assessment & First treatment $(n=97)$ & Switch $(n=102)$ & Total $(n=199)$ \\
\hline PDQ-D-20 & $-27.1(16.4)$ & $-33.9(15.8)$ & $-30.4(16.4)$ \\
QIDS-SR & $-11.1(5.0)$ & $-13.3(4.9)$ & $-12.2(5.0)$ \\
CGI-S & $-1.8(1.3)$ & $-2.2(1.0)$ & $-2.0(1.2)$ \\
WLQ, \% productivity loss & $-7.8(6.2)$ & $-10.1(5.4)$ & $-8.9(5.9)$ \\
WPAI, \% overall impairment & $-39.2(31.9)$ & $-42.9(34.4)$ & $-40.9(33.0)$ \\
SDS & $-13.2(8.4)$ & $-14.5(9.4)$ & $-13.9(8.9)$ \\
WHODAS & $-12.6(10.0)$ & $-14.0(10.2)$ & $-13.3(10.1)$ \\
DSST & $13.0(11.5)$ & $17.5(15.8)$ & $15.2(13.9)$ \\
GAD-7 & $-9.2(7.2)$ & $-9.9(6.5)$ & $-9.9(6.9)$ \\
\hline
\end{tabular}

Notes: *All changes are $p<0.001$ (paired $t$ test) versus baseline.

CGI-S, Clinical Global Impression-Severity; DSST, Digit Symbol Substitution Test; GAD-7, 7-item Generalized Anxiety Disorder Scale; PDQ-D-20, 20-item Perceived Deficits Questionnaire-Depression; QIDS-SR, Quick Inventory of Depressive Symptomatology-Self-Report; SD, standard deviation; SDS, Sheehan Disability Scale; WHODAS, 12-item World Health Organization Disability Assessment Schedule 2.0; WLQ, Work Limitations Questionnaire; WPAI, Work Productivity and Activity Impairment.

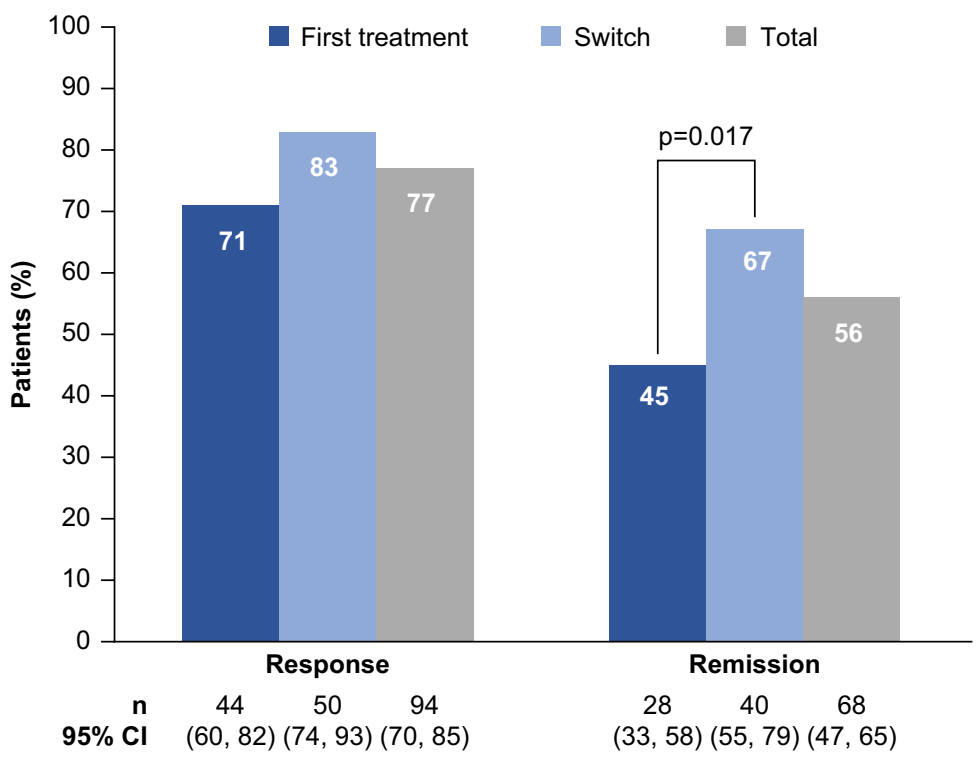

FIGURE 3. Rates of treatment response and remission after 52 weeks of vortioxetine treatment in the overall population and both subgroups (full analysis set, observed cases). $\mathrm{Cl}$, confidence interval; $\mathrm{n}$, number of patients who achieved response or remission; $\mathrm{p}$-value refers to the test for difference between the two groups (Fisher's exact test).

adjusted for age, sex, baseline PDQ-D-20, baseline WLQ productivity loss, disease duration, and disease severity at week 12 (primary study endpoint; $r=0.606 ; p<0.001$ ), and this association between PDQ-D-20 and WLQ productivity loss scores persisted at week $52(r=0.731 ; p<0.001$; Table 4). The correlations between changes in PDQ-D-20 scores and WLQ productivity loss were similar in firsttreatment and switch patient groups at both timepoints.

\section{SEM analysis}

Results of the SEM analyses are shown in Figure 5 and Supplementary Table 2. Patient-rated cognitive symptoms (PDQ-D-20 score), objective cognitive symptoms (DSST score), patient-rated depression severity (QIDS-SR score), and functional impairment (SDS total score or WLQ productivity loss score) were generally significantly dependent on the value of the previous assessment on the same scale at each timepoint over the 52 weeks of follow-up, ie, scores on each individual scale significantly predicted the subsequent score on the same scale (Supplementary Table 2).

Patient-rated cognitive symptoms (PDQ-D-20 score) at weeks 12 and 26 significantly predicted patient-rated functioning (SDS total score) at weeks 26 and 39 , 
TABLE 3. Pearson correlation coefficients between outcomes for changes from baseline to week 52 (full analysis set)

\begin{tabular}{|c|c|c|c|c|c|c|c|c|c|c|}
\hline Outcome & PDQ-D-20 & DSST & QIDS-SR & CGI-I: & CGI-S & $\mathrm{WLQ}^{+}$ & SDS & WPAI & WHODAS & GAD-7 \\
\hline PDQ-D-20 & 1.000 & & & & & & & & & \\
\hline DSST & $-0.252^{* *}$ & 1.000 & & & & & & & & \\
\hline QIDS-SR & $0.665^{* * *}$ & $-0.230 *$ & 1.000 & & & & & & & \\
\hline CGI-I & $0.396^{* * *}$ & 0.087 & $0.440^{* * *}$ & 1.000 & & & & & & \\
\hline CGI-S & $0.297^{* * *}$ & 0.120 & $0.336^{* * *}$ & $0.548^{* * *}$ & 1.000 & & & & & \\
\hline$W L Q^{\dagger}$ & $0.547^{* * *}$ & $-0.295^{* *}$ & $0.614^{* * *}$ & $0.299^{* *}$ & $0.314^{* * *}$ & 1.000 & & & & \\
\hline SDS & $0.589^{* * *}$ & $-0.198^{*}$ & $0.656^{* * *}$ & $0.361^{* * *}$ & $0.316^{* * *}$ & $0.695^{* * *}$ & 1.000 & & & \\
\hline WPAI & $0.380 * * *$ & $-0.231^{*}$ & $0.580^{* * *}$ & $0.326^{* * *}$ & $0.299 * *$ & $0.637^{* * *}$ & $0.674^{* * *}$ & 1.000 & & \\
\hline WHODAS & $0.506^{* * *}$ & -0.161 & $0.537^{* * *}$ & $0.340 * * *$ & $0.323^{* * *}$ & $0.656^{* * *}$ & $0.779 * * *$ & $0.599 * * *$ & 1.000 & \\
\hline GAD-7 & $0.548^{* * *}$ & $-0.224^{*}$ & $0.637^{* * *}$ & $0.300^{* * *}$ & $0.311^{* * *}$ & $0.617^{* * *}$ & $0.726^{* * *}$ & $0.578^{* * *}$ & $0.675^{* * *}$ & 1.000 \\
\hline
\end{tabular}

Notes: ${ }^{\dagger}$ WLQ productivity loss, \%. ${ }^{\ddagger} \mathrm{CGI}-\mathrm{I}$ scores at week 52 used for correlations. ${ }^{* * *} p \leq 0.001 .{ }^{* *} p \leq 0.01 .{ }^{*} p \leq 0.05$.

CGI-I, Clinical Global Impression-Improvement; CGI-S, Clinical Global Impression-Severity; DSST, Digit Symbol Substitution Test; GAD-7, 7-item Generalized Anxiety Disorder Scale; PDQ-D-20, 20-item Perceived Deficits Questionnaire-Depression; QIDS-SR, Quick Inventory of Depressive Symptomatology-Self-Report; SDS, Sheehan Disability Scale; WHODAS, 12-item World Health Organization Disability Assessment Schedule 2.0; WLQ, Work Limitations Questionnaire; WPAI, Work Productivity and Activity Impairment.

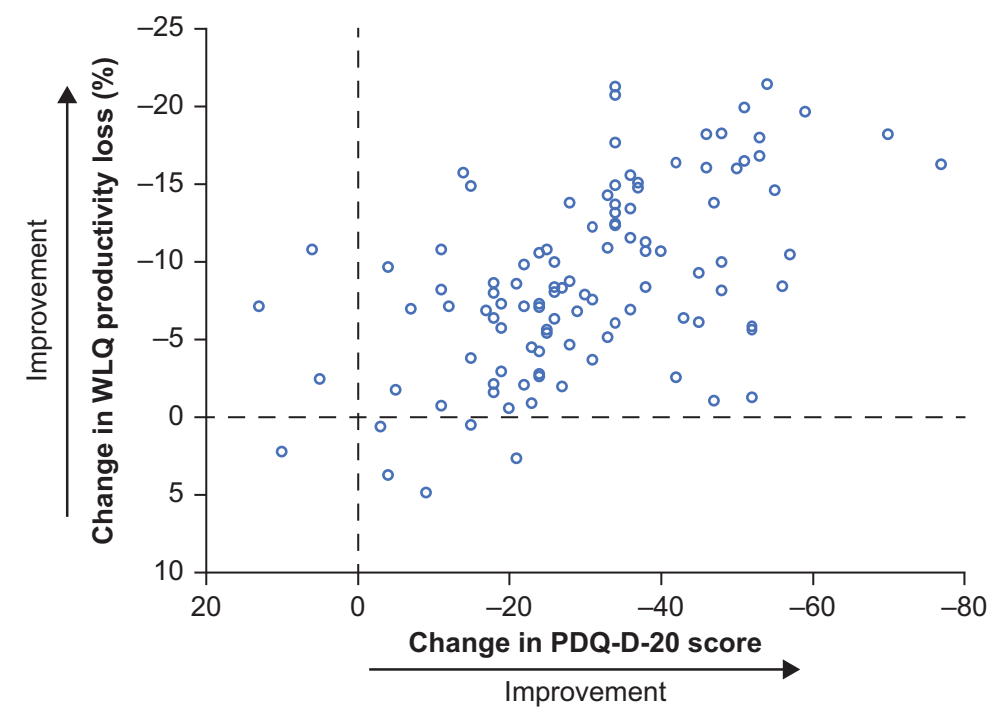

FIGURE 4. Scatter plot of individual changes from baseline to week 52 for PDQ-D20 and WLQ productivity loss scores (full analysis set, observed cases; $n$ = 107). PDQ-D-20, 20-item Perceived Deficits Questionnaire-Depression; WLQ, Work Limitations Questionnaire.

respectively (SRC, 0.22 and 0.27 ; both $p<0.05$ ). SRCs were adjusted for improvements in overall depressive symptoms, as the SEM included QIDS-SR scores. Patient-rated depression severity (QIDS-SR) scores did not significantly predict functioning outcomes (SDS or WLQ) at any subsequent timepoint; neither did PDQ-D-20 score predict WLQ productivity loss score at subsequent timepoints. Similarly, the objective cognitive performance measure (DSST) did not predict subjective measures of functioning (SDS or WLQ) at any subsequent timepoint.

PDQ-D-20 scores at weeks 12 and 39 significantly predicted QIDS-SR scores at the subsequent timepoints in the model including WLQ productivity loss (SRC, 0.20 and 0.27; $p<0.05$ and $p<0.01$, respectively). PDQ-D-20 score at week 26 also significantly predicted DSST score at week 39 in this model (SRC, $-0.22 ; p<0.05)$. In the model including SDS score, significant predictions were seen between PDQ-D-20 score at week 39 and QIDS-SR score at week 52 (SRC, 0.21), PDQ-D-20 score at week 26 and DSST score at week 39 (SRC, -0.22), and SDS score at week 26 and QIDS-SR score at week 39 (SRC, 0.22) (all $p<0.05$ ). Both models (including either WLQ or SDS as functioning outcomes) fitted the data well with root mean square error of approximation and comparative fit index values being indicative of good fit $(0.08$ and 0.95 , respectively, for the model including SDS total score; 0.08 and 0.94 , respectively, for the model including WLQ productivity loss). 
TABLE 4. Analysis of partial correlation* between changes from baseline at weeks 12 and 52 in PDQ-D-20 score and WLQ productivity loss (full analysis set, observed cases)

\begin{tabular}{|c|c|c|c|c|c|c|}
\hline \multirow[b]{2}{*}{ Group } & \multicolumn{3}{|c|}{ Week 12} & \multicolumn{3}{|c|}{ Week 52} \\
\hline & $N$ & $r$ & $p$-value & $\mathrm{n}$ & $r$ & $p$-value \\
\hline First treatment & 79 & 0.676 & $<0.001$ & 56 & 0.710 & $<0.001$ \\
\hline Switch & 75 & 0.515 & $<0.001$ & 51 & 0.788 & $<0.001$ \\
\hline Total & 154 & 0.606 & $<0.001$ & 107 & 0.731 & $<0.001$ \\
\hline
\end{tabular}

Notes: *Controlled for age, sex, baseline PDQ-D-20, baseline WLQ productivity loss, disease duration, and disease severity (baseline QIDS-SR, baseline CGI-S).

CGI-S, Clinical Global Impression-Severity; PDQ-D-20, 20-item Perceived Deficits Questionnaire-Depression; QIDS-SR, Quick Inventory of Depressive Symptomatology-Self-Report; WLQ, Work Limitations Questionnaire.

\section{Safety and tolerability}

Long-term treatment with vortioxetine was well tolerated. The most common treatment-emergent AEs were nausea (reported in $29.2 \%$ of treated patients), headache (11.9\%), insomnia $(9.1 \%)$, nasopharyngitis $(6.8 \%)$, anxiety $(6.4 \%)$, and dizziness $(5.9 \%)$ (Supplementary Table 3). No new safety signals were observed. At 52 weeks, 99 patients $(45.2 \%)$ had discontinued the study. The most common reason for study discontinuation was withdrawal of consent (42 patients, $19.2 \%$ ). Only 16 patients $(7.3 \%)$ discontinued the study due to an $\mathrm{AE}$ (9 in the first-treatment group and 7 in the switch group). Other reasons for discontinuation are shown in Supplementary Table 4.

\section{Discussion}

To our knowledge, this is the first study to examine the long-term effects of antidepressant therapy on cognition and functioning in working patients with MDD in a real-world setting. Patient demographic and disease characteristics at baseline were as expected for the general population presenting with MDD likely to be treated with antidepressants, supporting generalization of the study findings. Gainfully employed patients with MDD receiving vortioxetine in a real-life setting demonstrated clinically relevant improvements in mood, cognitive, and functional outcomes after continuous long-term treatment for up to 52 weeks. Work productivity improved over the 52 weeks of treatment by 8.9 percentage points on the WLQ productivity loss score, which ranges from 0 to $25 \%$ and assesses 4 dimensions of work productivity (time-management, physical demands, mental-interpersonal demands, and output demands). ${ }^{44}$

The highly significant association between improvements in cognitive symptoms (assessed using the PDQD-20) and workplace productivity loss previously reported after 12 weeks of vortioxetine treatment persisted at 52 weeks. ${ }^{40}$ These findings are in keeping with the results of a study in South Korea, in which MDD patients with greater severity of cognitive symptoms assessed by the PDQ-D-20 reported worse functional and work-related productivity outcomes, irrespective of depression severity. ${ }^{45}$ In a European study in patients with MDD who were either initiating or undergoing their first switch of antidepressant monotherapy (PERFORM), patient-reported cognitive symptoms (assessed using the shorter 5-item Perceived Deficit Questionnaire) were found to be independently associated with functional impairment, reduced work productivity, and lower quality of life throughout 2 years of follow-up. ${ }^{46,47}$

SEM analyses indicated that improvements in patientrated cognitive symptoms preceded long-term improvements in functioning outcomes, and that cognitive symptoms at weeks 12 and 26 significantly predicted functioning at the subsequent visits, even when adjusting for improvement in depressive symptoms (QIDS-SR). In the European PERFORM study, similar SEM analyses showed patient-reported cognitive symptoms to be an important determinant of both subsequent functional impairment and depression severity throughout 2 years of follow-up in patients with MDD who were either initiating or undergoing their first switch of antidepressant monotherapy. ${ }^{43}$ The present study found subjectively rated cognitive symptoms (PDQ-D-20) to be a stronger predictor than objective cognitive performance (DSST) of subsequent functioning outcomes, suggesting that PDQ-D-20 and DSST provide distinct assessments of cognitive function. Other studies have also found discrepancies between subjective and objective measures of cognition in patients with MDD. ${ }^{48-51}$ Collectively, such findings suggest that improvements in cognitive symptoms in patients with MDD may increase workplace productivity.

The significant improvements in both self-reported cognitive symptoms and cognitive performance assessed by the DSST seen in this study are consistent with results of previous studies of vortioxetine and reinforce these findings in a real-life setting. ${ }^{33-38}$ The significant improvements in work productivity seen in the present study during treatment with vortioxetine are consistent 
(a)

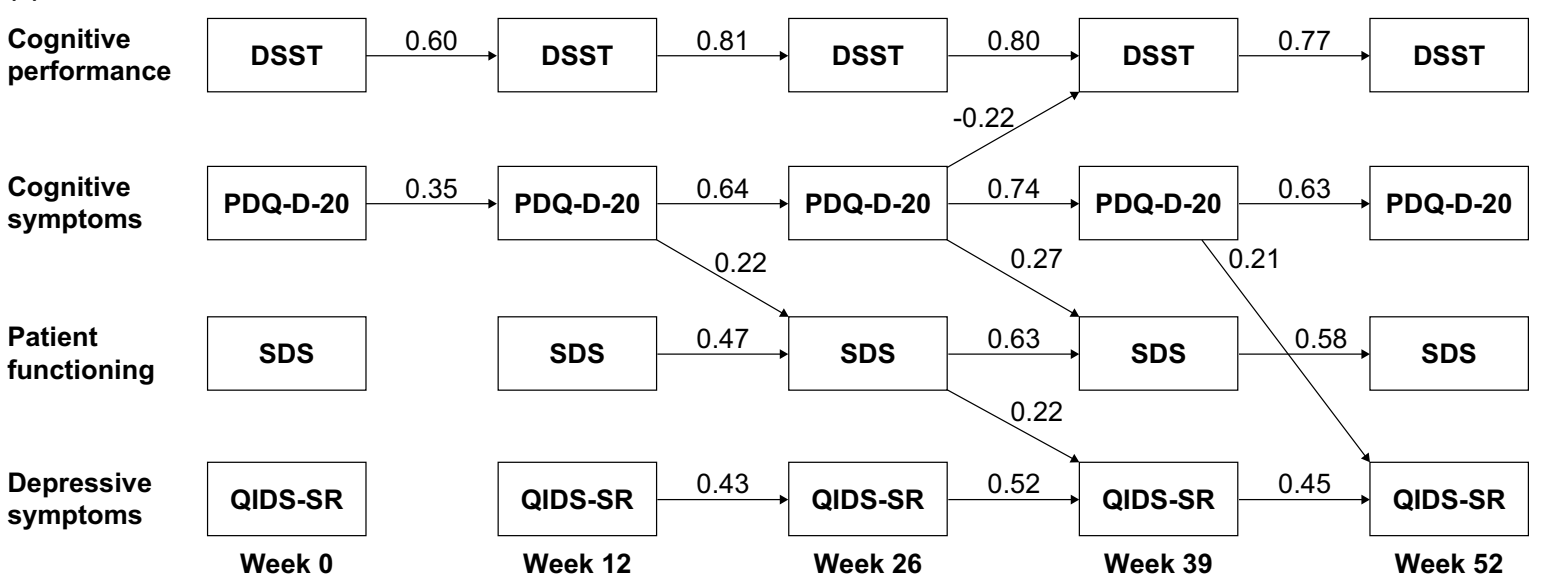

(b)

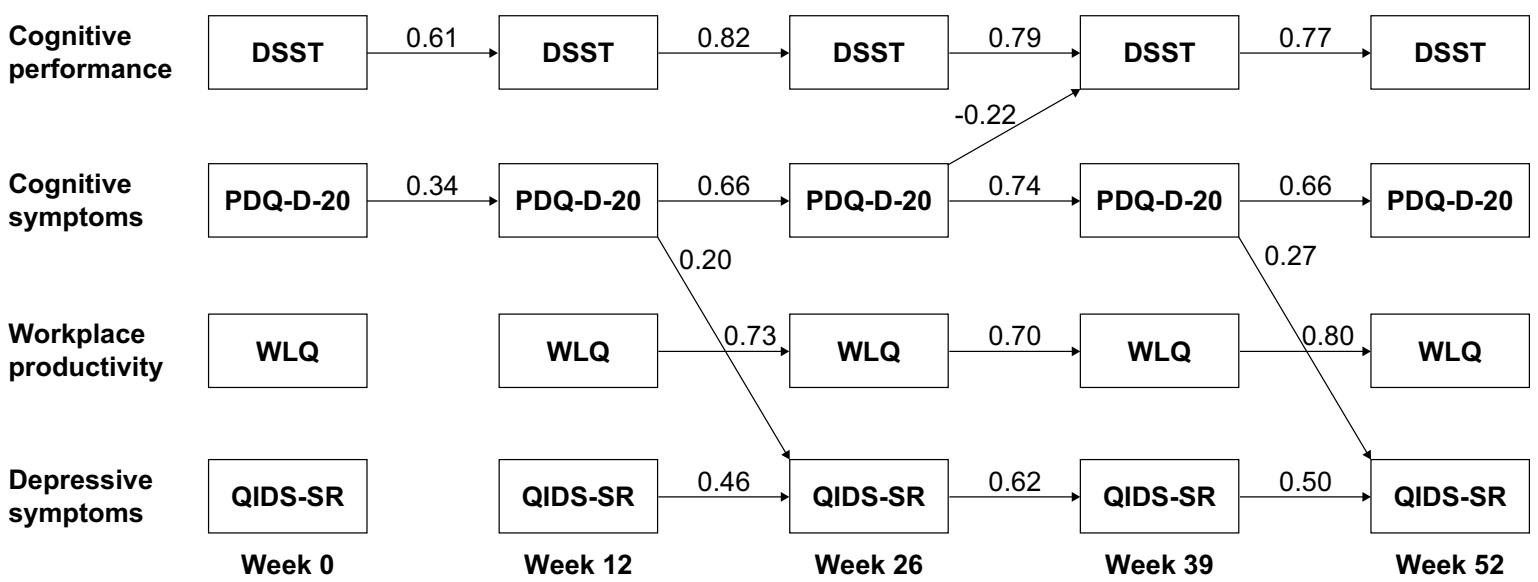

FIGURE 5. One-lag structural equations models of standardized scores for (a) DSST, PDQ-D-20, SDS, and QIDS-SR; and (b) DSST, PDQ-D-20, WLQ productivity loss, and QIDS-SR. DSST, Digit Symbol Substitution Test; PDQ, 20-item Perceived Deficits Questionnaire-Depression; QIDS-SR, Quick Inventory of Depressive Symptomatology-Self-Report; SDS, Sheehan Disability Scale; WLQ, Work Limitations Questionnaire. Standardized regression coefficients (SRC) shown on individual paths; paths with an SRC having an absolute value of less than 0.2 are omitted.

with the findings of the Combined Medications to Enhance Depression Outcomes (CO-MED) trial, which demonstrated that work productivity outcomes improve significantly with antidepressant treatment and that early changes in work productivity are significant predictors of long-term clinical course. ${ }^{52}$

In contrast to the results of the European PERFORM study, ${ }^{43}$ patient-rated depression severity (QIDS-SR) scores were not found to significantly predict functioning outcomes (SDS or WLQ) when simultaneously adjusting for improvements in cognitive symptom severity (PDQD-20 score) at any subsequent timepoint in the current SEM analyses. A reason for this result may be difference in sample sizes between the 2 studies. In the present study, unadjusted Pearson correlation coefficients did show significant associations between changes from baseline to week 52 for QIDS-SR scores and functioning outcomes (SDS and WLQ) as well as cognitive symptom severity (PDQ-D-20 score).

Depression and anxiety are frequently comorbid, with around $50 \%$ of patients with MDD also suffering from clinically significant levels of anxiety symptoms. ${ }^{53}$ Anxiety has been shown to contribute to increased rates of suicide, poor response to treatment, and increased risk of chronicity and recurrence in patients with depression. ${ }^{54,55}$ Significant improvement in the severity of anxiety symptoms was seen over the 52 weeks of vortioxetine treatment in the present study, from "severe" anxiety at baseline to "mild" at weeks 12 and 52 . A highly significant correlation between anxiety symptoms and workplace productivity was also observed at weeks 12 and 52 , highlighting a need for routine assessment and 
management of anxiety in patients with MDD for optimum outcomes.

High rates of treatment response and remission were seen after 52 weeks of vortioxetine treatment in this study. Overall, $77 \%$ of patients responded to treatment, and $56 \%$ of patients achieved remission after 52 weeks of vortioxetine. These rates are in keeping with those reported in a recent pooled analysis of data from 5 long-term, open-label extension studies of vortioxetine in patients with $\mathrm{MDD}{ }^{32}$ In that analysis, response (defined as $\geq 50 \%$ improvement in Montgomery Åsberg Depression Rating Scale [MADRS] score) was achieved in $75 \%$ of patients at week 52 and remission (defined as MADRS total score $\leq 10$ ) in $61 \%$. The high rate of remission achieved in switch patients in the present study is particularly noteworthy, as these patients had a statistically significantly longer duration of MDD than those receiving vortioxetine as first treatment for the current depressive episode (11.0 versus 5.6 years, respectively). The remission rate of $67 \%$ seen in switch patients after 52 weeks of vortioxetine treatment in this study is high when considered in context of the second-line remission rates reported by the Sequenced Treatment Alternatives to Relieve Depression (STAR $\left.{ }^{*} \mathrm{D}\right)$ study $^{56}$; in both realworld studies, remission was defined as QIDS-SR total score $\leq 5$. However, the high response and remission rates seen in patients switching to vortioxetine after inadequate response to a previous antidepressant in this real-world study are similar to those reported in previous randomized controlled studies of vortioxetine. ${ }^{31,57}$

Vortioxetine was well tolerated in this patient population. Safety and tolerability were consistent with previous reports of the short- and long-term tolerability of vortioxetine, ${ }^{58}$ and with the data reported in the Canadian product monograph for vortioxetine (Trintellix). ${ }^{59}$ Common AEs included nausea, headache, and insomnia, and few patients discontinued the study due to AEs. The overall discontinuation rate of $45 \%$ is not unexpected for a long-term study, and few patients discontinued due to AEs (7.3\%). This is similar to the overall discontinuation rate of $43 \%$ and a discontinuation rate due to AEs of $7.8 \%$ reported after 52 weeks of treatment with vortioxetine in a recent pooled analysis of data from 5 long-term, openlabel extension studies. ${ }^{32}$ Almost half of all patients who discontinued the AtWoRC study withdrew consent; as patients in this study were gainfully employed, it seems reasonable to assume that the need for regular study assessments over the 52 weeks of follow-up may have become burdensome.

A major strength of this study is that it was performed in a real-world setting with long-term follow-up. In addition, patient-reported outcome measures were used to assess disease severity and impact from the patient's own perspective. Use of patient-reported outcomes is in keeping with the general move toward increased patient involvement in treatment decisions, and awareness of the limitations of clinical symptom-based measures in assessing recovery from mental illness in a way that is meaningful to patients. ${ }^{60-63}$ Potential limitations include the open-label study design and the lack of a control group or active comparator; as such, the improvement observed was not controlled for any potential positive effect resulting from being included and assessed over time in the study. However, the AtWoRC study was undertaken primarily to assess the relationship between long-term changes in symptoms and workplace productivity in working patients with MDD treated with vortioxetine. The single-cohort study design was therefore appropriate, as the study was not designed to draw conclusions about the effectiveness of vortioxetine compared with other treatments.

\section{Conclusions}

In summary, results of the AtWoRC study demonstrate the long-term benefits of vortioxetine treatment in working patients with MDD in a real-world setting. Clinically relevant improvements in mood, anxiety and cognitive symptoms, work productivity, and functional outcomes were seen over the 52 weeks of treatment, as well as high rates of response and remission. A highly significant positive correlation was seen between changes in patientreported cognitive symptoms and workplace productivity after 12 and 52 weeks of vortioxetine treatment, with results of SEM analyses confirming that improvements in patient-rated cognitive symptoms predicted long-term improvements in functional outcomes even when adjusting for improvement in depressive symptoms. These findings suggest that treating cognitive symptoms is clinically important in order to achieve functional recovery in patients with MDD.

\section{Disclosures}

Pratap Chokka has received honoraria from serving on advisory boards with Allergan, Lundbeck, Janssen, Pfizer, Sunovion, Purdue, and Shire. He has also had speaking engagements with Allergan, Lundbeck, Janssen, Pfizer, Sunovion, Shire, and Purdue. He has received research grants from Lundbeck and Janssen. Joanna Bougie is an employee of Lundbeck Canada. Jean Proulx was an employee of Lundbeck Canada at the time the study was conducted. Anders Holmegaard Tvistholm and Anders Ettrup are employees of H. Lundbeck A/S. The authors declare no conflicts of interest in this work.

\section{Supplementary materials}

To view supplementary material for this article, please visit https://doi.org/10.1017/S1092852919000786 


\section{REFERENCES:}

1. American Psychiatric Association. Diagnostic and Statistical Manual of Mental Disorders. 5th ed. Washington, DC: American Psychiatric Association; 2013

2. IsHak WW, James DM, Mirocha J, et al. Patient-reported functioning in major depressive disorder. Ther Adv Chronic Dis. 2016;7(3): 160-169.

3. Beck A, Crain AL, Solberg LI, et al. Severity of depression and magnitude of productivity loss. Ann Fam Med. 2011;9(4):305-311.

4. Dewa CS, Thompson AH, Jacobs P. The association of treatment of depressive episodes and work productivity. Can J Psychiatry. 2011;56(12):743-750.

5. Jain G, Roy A, Harikrishnan V, Yu S, Dabbous O, Lawrence C. Patient-reported depression severity measured by the PHQ-9 and impact on work productivity: results from a survey of full-time employees in the United States. J Occup Environ Med. 2013;55(3):252-258.

6. Asami Y, Goren A, Okumura Y. Work productivity loss with depression, diagnosed and undiagnosed, among workers in an Internet-based survey conducted in Japan. J Occup Environ Med. 2015;57(1):105-110.

7. Uribe JM, Pinto DM, Vecino-Ortiz AI, Gómez-Restrepo C, Rondón M. Presenteeism, absenteeism, and lost work productivity among depressive patients from five cities of Colombia. Value Health Reg Issues. 2017;14:15-19.

8. Evans-Lacko S, Knapp M. Global patterns of workplace productivity for people with depression: absenteeism and presenteeism costs across eight diverse countries. Soc Psychiatry Psychiatr Epidemiol. 2016;51(11):1525-1537.

9. Stonebridge C, Sutherland G. Healthy Brains at Work. Estimating the Impact of Workplace Mental Health Benefits and Programs. Ottawa, Canada: The Conference Board of Canada; 1 September 2016.

10. Lam RW, McIntosh D, Wang J, et al. Canadian Network for Mood and Anxiety Treatments (CANMAT) 2016 clinical guidelines for the management of adults with major depressive disorder: Section 1. Disease burden and principles of care. Can J Psychiatry. 2016;61(9):510-523.

11. Greer TL, Kurian BT, Trivedi MH. Defining and measuring functional recovery from depression. CNS Drugs. 2010;24(4): 267-284.

12. Langlieb AM, Guico-Pabia CJ. Beyond symptomatic improvement: assessing real-world outcomes in patients with major depressive disorder. Prim Care Companion J Clin Psychiatry. 2010;12(2): e1-14.

13. McIntyre RS, Lee Y, Mansur RB. Treating to target in major depressive disorder: response to remission to functional recovery. CNS Spectr. 2015;20(Suppl 1):20-30.

14. Sheehan DV, Nakagome K, Asami Y, Pappadoulous EA, Boucher M. Restoring function in major depressive disorder: a systematic review. J Affect Disord. 2017;215:299-313.

15. Buist-Bouwman MA, Ormel J, de Graaf R, et al. Mediators of the association between depression and role functioning. Acta Psychiatr Scand. 2008;118(6):451-458.

16. Lee RS, Hermens DF, Porter MA, Redoblado-Hodge MA. A metaanalysis of cognitive deficits in first-episode major depressive disorder. J Affect Disord. 2012;140(2):113-124.

17. Iosifescu DV. The relation between mood, cognition and psychosocial functioning in psychiatric disorders. Eur Neuropsychopharmacol. 2012;22(Suppl 3):S499-S504.

18. Kennedy N, Foy K, Sherazi R, McDonough M, McKeon P. Long-term social functioning after depression treated by psychiatrists: a review. Bipolar Disord. 2007;9(1-2):25-37.

19. Trivedi MH, Morris DW, Wisniewski SR, et al. Increase in work productivity of depressed individuals with improvement in depressive symptom severity. Am J Psychiatry. 2013;170(6): 633-641.

20. Solomon DA, Leon AC, Endicott J, et al. Psychosocial impairment and recurrence of major depression. Compr Psychiatry. 2004;45(6):423-430.

21. Hardeveld F, Spijker J, De Graaf R, Nolen WA, Beekman AT. Prevalence and predictors of recurrence of major depressive disorder in the adult population. Acta Psychiatr Scand. 2010;122(3):184-191.

22. Hasselbalch BJ, Knorr U, Kessing LV. Cognitive impairment in the remitted state of unipolar depressive disorder: a systematic review. $J$ Affect Disord. 2011;134(1-3):20-31.

23. Goeldner C, Ballard TM, Knoflach F, Wichmann J, Gatti S, Umbricht D. Cognitive impairment in major depression and the mGlu2 receptor as a therapeutic target. Neuropharmacology. 2013;64:337-346

24. McIntyre RS, Cha DS, Soczynska JK, et al. Cognitive deficits and functional outcomes in major depressive disorder: determinants, substrates, and treatment interventions. Depress Anxiety. $2013 ; \mathbf{3 0}(6): 515-527$.

25. Bortolato B, Carvalho AF, McIntyre RS. Cognitive dysfunction in major depressive disorder: a state-of-the-art clinical review. CNS Neurol Disord Drug Targets. 2014;13(10):1804-1818.

26. Clark M, DiBenedetti D, Perez V. Cognitive dysfunction and work productivity in major depressive disorder. Expert Rev Pharmacoecon Outcomes Res. 2016;16(4):455-463.

27. Knight MJ, Baune BT. Cognitive dysfunction in major depressive disorder. Curr Opin Psychiatry. 2018;31(1):26-31.

28. McIntyre RS, Soczynska JZ, Woldeyohannes HO, et al. The impact of cognitive impairment on perceived workforce performance: results from the International Mood Disorders Collaborative Project. Compr Psychiatry. 2015;56:279-282.

29. Garnock-Jones KP. Vortioxetine: a review of its use in major depressive disorder. CNS Drugs. 2014;28(9):855-874.

30. Sanchez C, Asin KE, Artigas F. Vortioxetine, a novel antidepressant with multimodal activity: review of preclinical and clinical data. Pharmacol Ther. 2015;145:43-57.

31. Thase ME, Mahableshwarkar AR, Dragheim M, Loft H, Vieta E. A meta-analysis of randomized, placebo-controlled trials of vortioxetine for the treatment of major depressive disorder in adults. Eur Neuropsychopharmacol. 2016;26(6):979-993.

32. Vieta E, Loft H, Florea I. Effectiveness of long-term vortioxetine treatment of patients with major depressive disorder. Eur Neuropsychopharmacol. 2017;27(9):877-884.

33. McIntyre RS, Lophaven S, Olsen CK. A randomized, double-blind, placebo-controlled study of vortioxetine on cognitive function in depressed adults. Int J Neuropsychopharmacol. 2014;17(10): 1557-1567.

34. Mahableshwarkar AR, Zajecka J, Jacobson W, Chen Y, Keefe RS A randomized, placebo-controlled, active-reference, double-blind, flexible-dose study of the efficacy of vortioxetine on cognitive function in major depressive disorder. Neuropsychopharmacology. 2015;40(8):2025-2037.

35. Harrison JE, Lophaven S, Olsen CK. Which cognitive domains are improved by treatment with vortioxetine? Int $J$ Neuropsychopharmacol. 2016; 19(10): pyw054.

36. McIntyre RS, Harrison J, Loft H, Jacobson W, Olsen CK. The effects of vortioxetine on cognitive function in patients with major depressive disorder: a meta-analysis of three randomized controlled trials. Int J Neuropsychopharmacol. 2016; 19(10): pyw055.

37. McIntyre RS, Florea I, Tonnoir B, Loft H, Lam RW, Christensen MC. Efficacy of vortioxetine on cognitive functioning in working patients with major depressive disorder. J Clin Psychiatry. 2017;78(1): 115-121. 
38. Baune BT, Sluth LB, Olsen CK. The effects of vortioxetine on cognitive performance in working patients with major depressive disorder: a short-term, randomized, double-blind, exploratory study. $J$ Affect Disord. 2018;229:421-428.

39. Florea I, Loft H, Danchenko N, et al. The effect of vortioxetine on overall patient functioning in patients with major depressive disorder. Brain Behav. 2017;7(3):e00622.

40. Chokka P, Bougie J, Rampakakis E, Proulx J. Assessment in Work productivity and the Relationship with Cognitive symptoms (AtWoRC): primary analysis from a Canadian open-label study of vortioxetine in patients with major depressive disorder (MDD). CNS Spectr. In press. doi: 10.1017/S1092852918000913.

41. Mueller R, Hancock G. Best practices in structural equation modelling. In: Osborne J, ed. Best Practices in Qualitative Methods. Thousand Oaks, CA: Sage; 2008.

42. Kline RB. Principles and Practice of Structural Equation Modeling. New York: Guilford Press; 2011.

43. Larsen KG, Haro JM, Saragoussi D, Hammer-Helmich L. Depression severity and cognitive symptoms are independent predictors of functioning in patients with depression. Eur Neuropsychopharmacol. 2017;27(Suppl 4):s806.

44. Lerner D, Amick BC 3rd, Rogers WH, Malspeis S, Bungay K, Cynn D. The Work Limitations Questionnaire. Med Care. 2001; 39(1):72-85.

45. Kim JM, Chalem Y, di Nicola S, Hong JP, Won SH, Milea D. A crosssectional study of functional disabilities and perceived cognitive dysfunction in patients with major depressive disorder in South Korea: the PERFORM-K study. Psychiatry Res. 2016;239: 353-361.

46. Hammer-Helmich L, Haro JM, Jönsson B, et al. Functional impairment in patients with major depressive disorder: the 2-year PERFORM study. Neuropsychiatr Dis Treat. 2018;14:239-249.

47. Saragoussi D, Christensen MC, Hammer-Helmich L, Rive B, Touya M, Haro JM. Long-term follow-up on health-related quality of life in major depressive disorder: a 2-year European cohort study. Neuropsychiatr Dis Treat. 2018; 14:1339-1350.

48. Lam RW, Iverson GL, Evans VC, et al. The effects of desvenlafaxine on neurocognitive and work functioning in employed outpatients with major depressive disorder. J Affect Disord. 2016;203:55-61.

49. Cha DS, Carmona NE, Subramaniapillai M, et al. Cognitive impairment as measured by the THINC-integrated tool (THINC-it): Association with psychosocial function in major depressive disorder. J Affect Disord. 2017;222:14-20.

50. Srisurapanont M, Suttajit S, Eurviriyanukul K, Varnado P. Discrepancy between objective and subjective cognition in adults with major depressive disorder. Sci Rep. 2017;7(1):3901.
51. Fava M, Mahableshwarkar AR, Jacobson W, et al. What is the overlap between subjective and objective cognitive impairments in MDD? Ann Clin Psychiatry. 2018;30(3):176-184.

52. Jha MK, Minhajuddin A, Greer TL, Carmody T, Rush AJ, Trivedi MH Early improvement in work productivity predicts future clinical course in depressed outpatients: findings from the CO-MED trial. Am J Psychiatry. 2016;173(12):1196-1204.

53. Fava M, Rush AJ, Alpert JE, et al. Difference in treatment outcome in outpatients with anxious versus nonanxious depression: a $\mathrm{STAR}^{*} \mathrm{D}$ report. Am J Psychiatry. 2008;165(3):342-351.

54. Seo HJ, Jung YE, Kim TS, et al. Distinctive clinical characteristics and suicidal tendencies of patients with anxious depression. J Nerv Ment Dis. 2011;199(1):42-48.

55. Gaspersz R, Nawijn L, Lamers F, Penninx BWJH. Patients with anxious depression: overview of prevalence, pathophysiology and impact on course and treatment outcome. Curr Opin Psychiatry. 2018;31(1):17-25.

56. Rush AJ, Trivedi MH, Wisniewski SR, et al. Acute and longer-term outcomes in depressed outpatients requiring one or several treatment steps: a STAR ${ }^{*} \mathrm{D}$ report. Am J Psychiatry. 2006;163(11):1905-1917.

57. Montgomery SA, Nielsen RZ, Poulsen LH, Häggström L. A randomised, double-blind study in adults with major depressive disorder with an inadequate response to a single course of selective serotonin reuptake inhibitor or serotonin-noradrenaline reuptake inhibitor treatment switched to vortioxetine or agomelatine. Hum Psychopharmacol. 2014;29(5):470-482.

58. Baldwin DS, Chrones L, Florea I, et al. The safety and tolerability of vortioxetine: analysis of data from randomized placebo-controlled trials and open-label extension studies. J Psychopharmacol. 2016;30(3):242-252.

59. Lundbeck Canada Inc. TRINTELLIX ${ }^{\circledR}$ Product Monograph. https://www.lundbeck.com/upload/ca/en/files/pdf/pm/Trintellix. pdf. Accessed August 16, 2018.

60. Andresen R, Caputi P, Oades LG. Do clinical outcome measures assess consumer-defined recovery? Psychiatry Res. 2010;177(3): 309-317.

61. IsHak WW, Mirocha J, Pi S, et al. Patient-reported outcomes before and after treatment of major depressive disorder. Dialogues Clin Neurosci. 2014;16(2):171-183.

62. Slade M, Longden E. Empirical evidence about recovery and mental health. BMC Psychiatry. 2015;15:285

63. Oliveira-Maia AJ, Mendonça C, Pessoa MJ, Camacho M, Gago J. The mental health recovery measure can be used to assess aspects of both customer-based and service-based recovery in the context of severe mental illness. Front Psychol. 2016;7:1679. 